\title{
Residual life-based importance measures for predictive maintenance decision-making
}

\author{
Phuc Do ${ }^{1 *}$ and Christophe Bérenguer ${ }^{2}$ \\ ${ }^{1}$ Université de Lorraine, CRAN, UMR 7039, Campus Sciences, BP 70239, Vandoeuvre-les-Nancy, 54506, France \\ ${ }^{2}$ Univ. Grenoble Alpes, CNRS, Grenoble INP, GIPSA-lab, 38000 Grenoble, France
}

\begin{abstract}
Importance measures have been widely used as meaningful decision-aiding indicators in reliability engineering, risk management and maintenance optimization. However, few importance measures integrates the actual condition (working states or degradation levels) of components that dynamically evolves with time. This work develops a novel time-dependent importance measure defined as the capacity of a component (or group of components) to improve, when it is replaced, the system residual life. The proposed $I M_{M R L}$ measure can help to better prioritize a component or group of components regarding to its improvement ability in the system life time while considering the actual conditions of all components of the system. The originality and complementarity of the proposed measure when compared to existing importance measures is also investigated. The proposed importance measure is then extended to integrate the economic dimension of the maintenance decision, through the maintenance costs, the benefit gained by the maintenance operations and as well as the economic dependence between components. It is finally shown how the proposed $I M_{M R L}$ measure and its extension can "optimally" suggest a component or a group of several components for preventive maintenance decision-making, based on both the technical criterion (residual life of the system) and the economic aspects (benefit and costs). The use and the advantages of the proposed importance measure and its extension are illustrated on a 4-component system.
\end{abstract}

Keywords: Reliability, maintenance decision-making, importance measure, mean residual life, multicomponent system, economic dependence

\section{Introduction}

Within the framework of system engineering, importance measures are used to quantify the importance of a component or a group of components on the key performances of interest of a system, such as its reliability, its availability, its residual life, its productivity or its safety. These importance metrics can support decision-making to choose between several system upgrade options or design

\footnotetext{
${ }^{*}$ Corresponding author: phuc.do@univ-lorraine.fr
} 
modifications for correcting identified weaknesses, to select the most relevant maintenance actions or to identify and avoid operation bottlenecks. Different importance measures have been proposed in the field of reliability, risk and maintenance and they have been successfully used to solve many real-world problems, see [14] for a comprehensive overview of the classical importance measures and their applications. In risk analyses and reliability engineering, importance measures have been used for risk-informed decision-making [5,7] or for ranking components within a system for reliability improvement purposes $[1,2,4,10]$. Recently, several applications of importance measures have been developed aiming at maintenance optimization and spare parts management [17, 21, 24]. For example, for complex multi-component systems, a maintenance optimization approach based on the Birnbaum structural importance measure has been developed in [16, 21]. [3] has proposed to resort to a differential importance measure for inventory management. More recent works have addressed the issue of basing the preventive maintenance decisions on importance metrics of the maintained system's components $[10,24,26]$.

With the aim of optimizing condition-based or predictive maintenance decisions on a system, the available information on the actual condition of components (states or degradation levels) is obviously a critical input to the decision process and should be considered in the synthesis of decision-aiding indicators. Following this idea, a data-driven framework integrating the deterioration monitoring information has been recently introduced to identify the critical components of a complex technical infrastructure in [15] and a new importance measure, called condition-based importance measure, has been recently proposed in [10]. It relies on the quantification of the reliability improvement for a given finite horizon $(t, t+u)$ brought by a component replacement and it integrates the available information on the actual condition of all the system components at decision time point $t$. This importance measure proved to be relevant for ranking the system components with respect to a reliability metric on a finite horizon. However, it cannot be directly applied for an infinite horizon reliability metric such as the system residual life (or the remaining useful life) that is nevertheless considered as an important reliability metric for various decision-making, see for instance [12, 19, 22]. To answer this gap, this work proposes a novel importance measure based on the system residual life, namely $I M_{M R L}$ measure, that can be used to rank components/groups of components with respect to their ability to improve the system residual life considering all the available information of the actual conditions of all components at the decision time point. To highlight the originality and complementarity of the proposed $I M_{M R L}$ measure when compared to existing ones, some direct links between these already existing measures and the proposed one are also highlighted and investigated.

Moreover, when implementing maintenance operations in practice, an economic dependence often arises : in this case, the joint maintenance of several components turns out to be cheaper than maintaining separately the components. Such an economic dependence in maintenance should be integrated appropriately in the maintenance decision-making. However, to the best of our knowledge, 
very few existing importance measures designed to support maintenance decisions actually integrate this kind of economic dependence between components. To fill this second gap, an extension of the proposed $I M_{M R L}$ measure is thus proposed in this work to take into account the economic aspects related to maintenance, i.e. costs incurred by maintenance actions, with the economic dependence, and benefit gained from maintained system operation. The proposed $I M_{M R L}$ measure and its extension allow ranking and selecting the best candidate component or group of components for maintenance according to both a technical criterion (residual life of the system) and/or economic considerations (benefit and cost).

In summary, the contribution of the work presented in this paper is twofold: (i) it first proposes a component importance measure elaborated from the estimated improvement in the mean system residual life brought by the as-good-as new replacement of the component, given the available information on the actual condition of all the system components ; (ii) the proposed importance measure is then extended to integrate economic aspects related to maintenance costs and system profit. This paper develops an extended version of the work presented in [11].

The remainder of this paper is organized as follows. Section 2 is devoted to the presentation of the main modelling assumptions of this work. The different considered levels for the monitoring information and the mean residual life are presented and discussed. In addition, the impact of the information levels on the estimated system residual life is also highlighted. The proposed $I M_{M R L}$ measure is introduced in Section 3. The link with two existing importance measures in different contexts related to given components' information levels is discussed. In Section 4, an extension of $I M_{M R L}$ measure integrating economic aspects is developed. The economic dependence between components is also studied. To illustrate the use and the advantages of $I M_{M R L}$ measure and its extension, Section 5 presents a numerical example of a 4-component system. In addition, various numerical studies are herein analyzed and discussed. Finally, the conclusions drawn from this work and some perspectives are presented in the last section. 


\section{Notation}
$a, b$
economic dependence factors
$C^{i}$
maintenance cost of component $i$
$d^{i}$
duration of a replacement for component $i$
$\left(X_{t}^{i}\right)_{t \geq 0}$
stochastic degradation process component $i$ over time $t$
$x_{t}^{i}$
degradation level of component $i$ at time $t$
$L^{i}$
failure threshold of component $i$
$\mathfrak{Z}_{t}^{i}, \mathfrak{S}_{t}$
type of available information on the condition of component $i$ and the system at $t$ respect
$\left.\mathbf{Z}_{t}=\left(\mathfrak{Z}_{t}^{1}, \mathfrak{Z}_{t}^{2}\right), \ldots \mathfrak{Z}_{t}^{n}\right)$
information about all components of the system at time $t$
$r^{i}\left(t+u \mid \mathfrak{Z}_{t}^{i}\right)$
conditional reliability of component $i$
$R(t)$
reliability of the system
$R\left(t+u \mid \mathbf{Z}_{t}\right)$
reliability of the system given the condition/information $\mathbf{Z}_{t}$
$f_{\tau_{i}}(u)$
probability density function for the failure time of component $i$
$f_{\tau_{i}}(u)$
probability density function for the failure time of component $i$
$f_{X_{t}^{i}}(u)$
probability density function for the degradation level of component $i$ at time $t$
$\operatorname{MRL}\left(t \mid \mathbf{Z}_{t}\right)$
Mean residual life of the system at time $t$ given the information $\mathbf{Z}_{t}$

\section{Modelling framework}

\subsection{System modelling assumptions}

We consider a coherent structured system consisting of $n$ non-identical components that are organised according to a structure assumed to be known (e.g., series, parallels or mix, etc.). Each component may fail because of an underlying random ageing phenomenon or a stochastic degradation process. The failures of the components are assumed to be independent.

Only perfect maintenance actions or replacements are assumed to be implementable on the system, i.e., performing a maintenance action on a component brings the maintained component into an "asgood-as new" state. In addition, each maintenance action incurs a maintenance cost.

\subsection{Component failure modelling and system reliability assessment}

Component failure modelling In reliability engineering, the reliability of an entity (component or system) is defined as the probability that the entity performs its mission without any failure until time $t$. To assess the reliability of a component, two main approaches are classically used: lifetime models or degradation-based failure models [17, 19]. The use of either model depends on the level of information available on the state of the considered component. Lifetime models are suitable to describe the situation where the information on the component degradation levels is not available for technical and/or economical reasons (e.g., it is not allowed or impossible to inspect in-depth the component 
to reveal its degradation level; its inspection operation is too expensive or the component is highly reliable and its inspection is not considered to be worthwhile). In this situation, the component is only known to be working or failed. On the contrary, when the degradation level of the component can be monitored more closely and finely, a degradation-based failure model based on a stochastic process is more informative and is often preferable. Accordingly, the two following cases can be considered:

- Components whose failure behavior is described by a lifetime model are referred to as "ageing components". The reliability of the ageing component $i$ can be written

$$
r^{i}(t)=\mathbb{P}\left(\tau_{i}>t\right)=1-\int_{0}^{t} f_{\tau_{i}}(x) d x
$$

where $\tau_{i}$ is the time to failure of component $i$ and follows a probability distribution with probability density function $f_{\tau_{i}}(x)$. As an example, the Weibull distribution which has been widely used in the literature for reliability modeling at component level [19], is used for reliability assessment of certain components in this work. Of course, other probability laws could be used in a similar manner. A detailed description of the Weibull distribution is given in Appendix A.

- Components that gradually degrades with wear and tear, and whose degradation can be monitored are referred to as "degrading components". Assuming that the degradation evolution of the degrading component $i$ is described by a stochastic process $X_{t}^{i}$, its reliability can be then written as:

$$
r^{i}(t)=\mathbb{P}\left(X_{t}^{i}<L^{i}\right)=\int_{0}^{L^{i}} f_{X_{t}^{i}}(x) d x,
$$

where $L^{i}$ is the degradation failure threshold above which the component $i$ is considered as failed (i.e., when $X_{t}^{i} \geq L^{i}$, the component $i$ is failed) and $f_{X_{t}^{i}}(u)$ is the probability density function of the degradation level of component $i$ at time $t$. In the literature, different degradation stochastic processes are available such as gamma, Wiener, etc $[19,20]$. In this work, a gamma stochastic process is used as an example for reliability assessment of degrading components. A detailed description is given in Appendix B.

System reliability Because the components failures are assumed to be statistically independent, the system reliability can be computed from the components' reliabilities using the system reliability structure function. More precisely, let $R(t)$ be the system reliability. $R(t)$ is a function of the reliabilities of all the system components $r^{i}(t)$ with $i=1,2, \ldots n$ :

$$
R(t)=\varphi\left(r^{1}(t), r^{2}(t), \ldots, r^{n}(t)\right) .
$$

The structure function $\varphi($.) can be obtained from the minimal path sets or the minimal cut sets of the system [19]. As an example, the reliability of a series structure of two component is given by Eq.(11) and the reliability of four-component system with a mixed structure is provided in Section 5 . 


\subsection{Level of information and mean residual life}

\subsubsection{Level of information and conditional reliability assessment}

Let $\mathfrak{S}_{t}$ be the state of the system at time $t$. As only binary states are considered at the system level, $\mathfrak{S}_{t}$ can be expressed as follows

$$
\mathfrak{S}_{t}= \begin{cases}1 & \text { if the system is functioning at time } t \\ 0 & \text { otherwise. }\end{cases}
$$

It is assumed that at initial time, $t=0$, the system is new $\mathfrak{S}_{0}=1$. At time $t$, two cases can be distinguished:

- if the system is in a failed state, $\mathfrak{S}_{t}=0: R\left(t+u \mid \mathfrak{S}_{t}=0\right)=0$.

- if the system is functioning at time $t, \mathfrak{S}_{t}=1$, the predicted conditional reliability of the system at time $t+u$ (with $u>0$ ) can be computed using the available information at time $t$ from Eq.(3) by replacing the components reliability $r^{i}(t)$, with $i=1,2, \ldots, n$, by the components conditional reliability $r^{i}\left(t+u \mid \mathfrak{Z}_{t}^{i}\right)$ where $\mathfrak{Z}_{t}^{i}$ represents the available information about the state of component $i$ at time $t$.

It should be noticed that the available information about the state of components is crucial for predicting the components' reliability. For degrading components, four levels of information can be considered:

$\mathfrak{Z}_{t}^{i}= \begin{cases}0 & \text { if component } i \text { is known to be in a failed state at time } t \\ 1 & \text { if component } i \text { is known to be functioning at time } t \text { but its degradation level is not available; } \\ 2 & \text { if component } i \text { is known to be functioning and its degradation level is measured at time } t \\ 3 & \text { if component } i \text { is known to be replaced by a new component at time } t\end{cases}$ Accordingly, given the information level available at time $t$ on a component state, the conditional reliability of the component can be calculated by distinguishing the following 4 cases :

- If component $i$ is failed at time $t, \mathfrak{Z}_{t}^{i}=0$, its conditional reliability at $t+u$ is equal to zero (no replacement is assumed between $t$ and $t+u): r^{i}\left(t+u \mid \mathfrak{Z}_{t}^{i}=0\right)=0$;

- If component $i$ is still working at time $t$ but no information on its degradation level is available (because of a technical or economical reason), $\mathfrak{Z}_{t}^{i}=1$, its conditional reliability at $t+u$ writes

$$
r^{i}\left(t+u \mid \mathfrak{Z}_{t}^{i}=1\right)=\frac{r^{i}(t+u)}{r^{i}(t)}
$$

It is worth noting that in the case of an exponential lifetime law for component $i$, i.e. its failure rate is time-independent, its conditional reliability can be calculated by $r^{i}\left(t+u \mid \mathfrak{Z}_{t}^{i}=1\right)=r^{i}(u)$, which means that component $i$ can be considered as new if it is known that it has survived time $t$, and thus no preventive maintenance is required. 
- If component $i$ is still working and its monitored degradation level is available at time $t, \mathfrak{Z}_{t}^{i}=2$, its conditional reliability at $t+u$ is then given by

$$
\begin{aligned}
r^{i}\left(t+u \mid \mathfrak{Z}_{t}^{i}=2\right) & =\mathbb{P}\left(X_{t+u}^{i}<Z_{i} \mid X_{t}^{i}=x_{t}^{i}\right) \\
& =\int_{x_{t}^{i}}^{Z_{i}} f_{X_{t+u}^{i}}(x) d x
\end{aligned}
$$

where $X_{t}^{i}=x_{t}^{i}$ denotes the degradation level of component $i$ at time $t$.

- If component $i$ is replaced by a new one at time $t, \mathfrak{Z}_{t}^{i}=3$, its conditional reliability writes simply

$$
r^{i}\left(t+u \mid \mathfrak{Z}_{t}^{i}=3\right)=r^{i}(u)
$$

The conditional reliability assessment of an ageing component remains similar to that of a degrading component, noting however that for an ageing component $i$, according to the definition used in this work, only three levels of information on the component state are considered, i.e., $\mathfrak{Z}_{t}^{i}$ can be 0,1 or 3 (no degradation level information is ever available for degrading components).

It must be noticed that for industrial systems, the replacement of component $i$ may require disassembling other components, this phenomenon is called structural dependence [18]. The disassembly operations may affect the failure/degradation processes of certain disassembled components. Such a structural dependence is not considered in this work, but for further discussion on the disassembly impacts, the interested reader can consult [8].

\subsubsection{Mean residual life}

The residual life (or remaining useful life) of a system is an important reliability metric of the system since it provides the information about the duration left before the system fails given the condition of its components at time $t[6,19,25]$. Even though the full probabilistic characterization of the random resiudal life contains more information, the mean residual life (MRL) can still be used as an appropriate reliability metric for various decision-making $[12,19,22]$. Indeed, the mean residual life of a system at time $t$ is defined as the mean time left before the system failure.

Let $\left.\mathbf{Z}_{t}=\left(\mathfrak{Z}_{t}^{1}, \mathfrak{Z}_{t}^{2}\right), \ldots \mathfrak{Z}_{t}^{n}\right)$ be the vector representing the information about all components of the system at time $t$. The mean residual life of a system at time $t$ is mathematically expressed as follows:

$$
\operatorname{MRL}\left(t \mid \mathbf{Z}_{t}\right)=\int_{0}^{\infty} R\left(t+u \mid \mathbf{Z}_{t}\right) d u
$$

Obviously $M R L\left(t \mid \mathbf{Z}_{t}\right)$ depends not only on the time $t$ but also on level of the available information on the components' state at time $t$. In addition, $M R L\left(t \mid \mathbf{Z}_{t}\right)$ has the following properties:

- $M R L\left(t \mid \mathbf{Z}_{t}\right) \geq 0$ and $M R L\left(t \mid \mathbf{Z}_{t}\right)=0$ if the system fails at time $t$, i.e., $\mathfrak{S}_{t}=0$; 
- At the initial time point $t=0$, all the components are new $\left(\mathfrak{Z}_{t}^{i}=3\right.$ with $\left.i=1, \ldots, n\right)$, we get:

$$
\operatorname{MRL}\left(0 \mid \mathfrak{Z}_{0}^{1}=3, \mathfrak{Z}_{0}^{2}=3, \ldots, \mathfrak{Z}_{0}^{n}=3\right)=\int_{0}^{\infty} R(u) d u=M T T F,
$$

where $M T T F$ represents the mean time to failure of the system [19];

- For a coherent system, without any maintenance intervention $M R L\left(t \mid \mathbf{Z}_{t}\right)$ is a decreasing function over time, i.e., $M T T F \geq M R L\left(t_{1} \mid \mathbf{Z}_{t_{1}}\right) \geq M R L\left(t_{2} \mid \mathbf{Z}_{t_{2}}\right)$ with $t_{1} \leq t_{2}$;

- If all the components of the system are replaced simultaneously at time $t$, i.e. the system is renewed at time $t$, we get:

$$
\operatorname{MRL}\left(t \mid \mathfrak{Z}_{t}^{1}=3, \mathfrak{Z}_{t}^{2}=3, \ldots, \mathfrak{Z}_{t}^{n}=3\right)=\operatorname{MTTF}
$$

Assume that at time $t$, only component $i$ is replaced, the mean residual life of a system is then:

$$
\operatorname{MRL}\left(t \mid \mathbf{Z}_{t}, \mathfrak{Z}_{t}^{i}=3\right)=\int_{0}^{\infty} R\left(t+u \mid \mathbf{Z}_{t}, \mathfrak{Z}_{t}^{i}=3\right) d u
$$

Example 1 For illustration purposes, consider the simple example of a 2-component series system. Both components of the system, $C 1$ and $C 2$, are assumed to undergo a degradation process that can be described by a gamma process with a shape and a scale parameter $\left(\alpha^{i}, \beta^{i}\right)$ with $i=1,2$ (see Appendix B for a detailed description of the gamma process). Both components fail as soon as their degradation level reach their failure threshold, resp $L^{1}=L^{2}=100$. The system reliability block diagram and the values of the parameters are given in Figure 1.

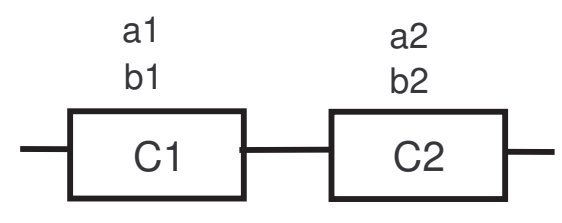

Figure 1: A 2-component system.

The reliability of the system is obtained readily as:

$$
R(t)=r^{1}(t) \cdot r^{2}(t)
$$

To illustrate the impact on the estimated mean residual life of the level of the available information on the components states, we consider the three following cases:

- Both components are monitored and their degradation state is measured and available at each time unit, i.e. $\mathfrak{Z}_{t}^{1}=\mathfrak{Z}_{t}^{2}=2$;

- Component $\mathrm{C} 2$ is monitored and its degradation state is measured and available at each time unit, however no inspection operation is ever realised on component $\mathrm{C} 1$ due to a technical or economical reason, i.e. $\mathfrak{Z}_{t}^{1}=1, \mathfrak{Z}_{t}^{2}=2$; 
- Neither of the two components C1 and C2 is monitored and their state of degradation remains unknown, i.e. $\mathfrak{Z}_{t}^{1}=\mathfrak{Z}_{t}^{2}=1$.

Figure 2 shows an example of the degradation paths of each component and the estimated mean residual life $M R L\left(t \mid \mathbf{Z}_{t}\right)$ of the system according to the three mentioned cases of information level. This example illustrates the fact that the estimated mean residual life $M R L\left(t \mid \mathbf{Z}_{t}\right)$ depends on the information levels of both components $\mathrm{C} 1$ and $\mathrm{C} 2$. Ignoring the degradation levels of one or two components may lead to a significant error in the evaluation of $M R L\left(t \mid \mathbf{Z}_{t}\right)$.
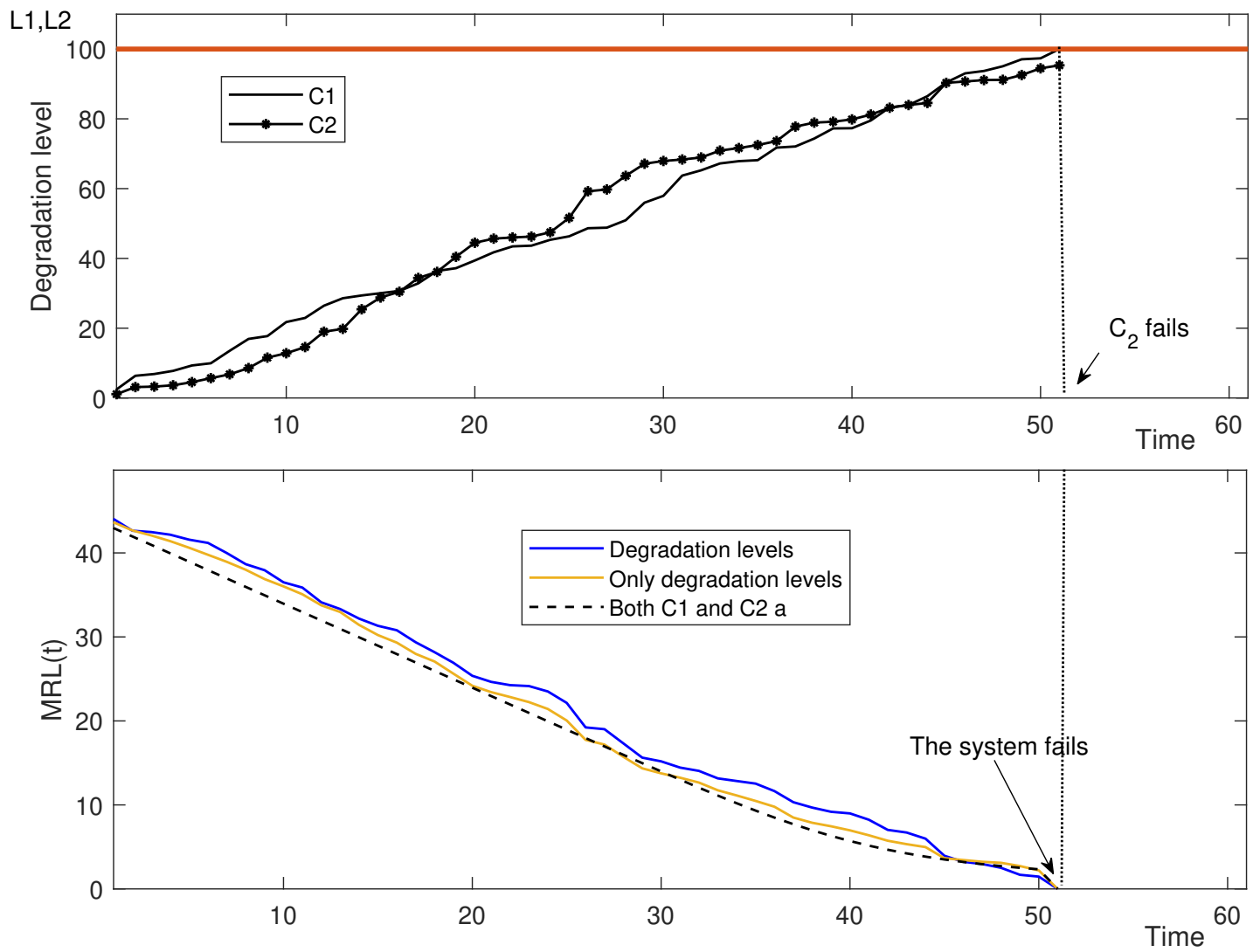

Figure 2: Components' degradation paths of a two-component system and the evolution of the estimated system mean residual life for different levels of information.

As the mean residual life is an important reliability metric of the system and as it allows to embed in its estimation process different levels of information, in this study it is used to define a novel importance measures. The detailed discussion is given in the following section. 


\section{Importance measures based on the mean residual life}

\subsection{Definition of $I M_{M R L}$ measure}

An importance measure based on the mean residual life can be defined as follows for component $i$ :

$$
I M_{M R L}^{i}(t)=M R L\left(t \mid \mathbf{Z}_{t}, \mathfrak{Z}_{t}^{i}=3\right)-M R L\left(t \mid \mathbf{Z}_{t}\right) .
$$

$I M_{M R L}^{i}(t)$ quantifies the improvement in the system mean residual life time brought by the replacement of component $i$ at time $t$, given the available information of the actual condition of all the other system components. The $I M_{M R L}$ measure is characterized by the following properties:

- For a coherent system $M R L\left(t \mid \mathbf{Z}_{t}, \mathfrak{Z}_{t}^{i}=3\right) \geq M R L\left(t \mid \mathbf{Z}_{t}\right)$, and $I M_{M R L}^{i}(t)$ is thus non negative and $I M_{M R L}^{i}(t) \leq M T T F$ :

$-I M_{M R L}^{i}(0)=0$

- IM $M_{M R L}^{i}(t)=0$ if component $i$ is known to be still working at time $t$ and its failure rate is time-independent. This means that the replacement of component $i$ does not have any impact on the residual life time of the system, which leads to the well-known result that it is not necessary to replace component $i$ with a constant failure rate if it is kown to be still working at time $t$;

- IM $M_{M R L}^{i}(t)=M T T F$ if the system is composed of a single component $(n=1)$ and the component is in a failed state at time $t$.

- $I M_{M R L}^{i}(t)$ depends on the available information on the actual state or degradation level of all the components at time $t$;

- Finally, $I M_{M R L}^{i}(t)$ is time-dependent if the failure/degradation process of one or several components is time-dependent.

From Eq.(7) and Eq.(10), $I M_{M R L}^{i}(t)$ can be written as:

$$
\begin{aligned}
I M_{M R L}^{i}(t) & =\int_{0}^{\infty} R\left(t+u \mid \mathbf{Z}_{t}, \mathfrak{Z}_{t}^{i}=3\right) d u-\int_{0}^{\infty} R\left(t+u \mid \mathbf{Z}_{t}\right) d u \\
& =\int_{0}^{\infty}\left[R\left(t+u \mid \mathbf{Z}_{t}, \mathfrak{Z}_{t}^{i}=3\right)-R\left(t+u \mid \mathbf{Z}_{t}\right)\right] d u
\end{aligned}
$$

In the purpose of condition-based maintenance decision-making, as $I M_{M R L}$ measure allows integrating the available information on the current actual condition (state or degradation level) of all components, it can be a relevant indicator to identify the most important component that should be replaced, at given time $t$, to improve the system residual life. 
Example 1 (continued) Reconsider now the example of 2-component system shown in Figure 1 and assume that the system is still functioning at time $t$, i.e. both components have survived $t, \mathfrak{Z}_{t}^{i} \neq 0$ with $i=1,2$. The system mean residual life in the absence of any maintenance action at time $t$ is given by:

$$
\operatorname{MLR}\left(t \mid \mathfrak{Z}_{t}^{1} \neq, \mathfrak{Z}_{t}^{2} \neq 0\right)=\int_{0}^{\infty} r^{1}\left(t+u \mid \mathfrak{Z}_{t}^{1} \neq 0\right) \cdot r^{2}\left(t+u \mid \mathfrak{Z}_{t}^{2} \neq 0\right) d u
$$

The $I M_{M R L}$ measure of component $i(i=1,2)$ writes as:

$$
I M_{M R L}^{i}(t)=\int_{0}^{\infty} r^{3-i}\left(t+u \mid \mathfrak{Z}_{t}^{3-i} \neq 0\right) \cdot\left[r^{i}(u)-r^{i}\left(t+u \mid \mathfrak{Z}_{t}^{i} \neq 0\right)\right] d u .
$$

Two different cases can be distinguished depending on the level of the available information on both components at time $t$ :

Case 1 Both components are functioning at time $t$ but their degradation levels are not available, i.e. $\mathfrak{Z}_{t}^{1}=\mathfrak{Z}_{t}^{2}=1$. In this case, Table 1 shows the value of the $I M_{M R L}$ measure for each component obtained using Eq.(14) and Eq.(4) : component C2 turns out to be more important than C1, which leads to conclude that the replacement of $\mathrm{C} 2$ is more effective than the replacement of $\mathrm{C} 1$ to improve the system mean residual life.

Case 2 Both components $\mathrm{C} 1$ and $\mathrm{C} 2$ are monitored and the information on their degradation level is available, i.e. $\mathfrak{Z}_{t}^{1}=\mathfrak{Z}_{t}^{2}=2$. Both components being subject to a stochastic degradation process, the degradation level of each component at time can be random and two different degradation path realizations are considered in this example, refered to as respectively Case $2 \mathrm{a}$ and $2 \mathrm{~b}$. The different degradation levels of both components at time $t=10$ in each case (2a and $2 \mathrm{~b}$ ) are reported in Table 1 and are used to compute in each case the value of $I M_{M R L}$ measure for both components at time $t=10$, using Eq.(14) and Eq.(5). For the first realization of the degradation path (Case 2a), $I M_{M R L}^{1}(t)$ is greater than $I M_{M R L}^{2}(t)$ and component $\mathrm{C} 1$ is thus more important than component $\mathrm{C} 2$ in improving the system mean residual life. It should be noticed that this importance ranking is not the same as the one observed when the components' degradation levels are unknown. On the contrary, for the second realization of the degradation path (case 2b), $I M_{M R L}^{1}(t)<I M_{M R L}^{2}(t)$, i.e. C2 turns out to be more important than C1.

\begin{tabular}{|c|cc|cc|c|c|cc|}
\cline { 2 - 9 } \multicolumn{1}{c|}{} & \multicolumn{3}{c|}{ Information at $t=10$} & \multicolumn{2}{c|}{$I M_{M R L}$ measure at $t=10$} & \multicolumn{2}{c|}{ Ranking } \\
\cline { 2 - 9 } \multicolumn{1}{c|}{} & $\mathfrak{Z}_{t}^{1}$ & $X_{t}^{1}$ & $\mathfrak{Z}_{t}^{2}$ & $X_{t}^{2}$ & $I M_{M R L}^{1}(t)$ & $I M_{M R L}^{2}(t)$ & C1 & C2 \\
\hline Case 1 & 1 & - & 1 & - & 2.69 & 3.06 & 2 & 1 \\
\hline Case 2a & 2 & 21.76 & 2 & 12.82 & 3.42 & 1.35 & 1 & 2 \\
Case 2b & 2 & 15.91 & 2 & 22.16 & 1.33 & 4.19 & 2 & 1 \\
\hline
\end{tabular}

Table 1: Example of $I M_{M R L}$ measure and components ranking. 
The evolution of the $I M_{M R L}$ measure for both components as a function of time $t$ in both considered cases (1 and 2a) are sketched in Figure 3. Because $I M_{M R L}$ measures depend on the information levels available for both components and because the actual degradation paths of each component evolves stochastically with time, $I M_{M R L}$ measures change stochastically with time in Case 2a (degradation levels of both components are measured). It should be noticed that $I M_{M R L}$ measure of each individual component is evaluated based on the degradation paths of both components that are shown in Figure 2. In Case 1 (degradation levels of both components are unknown), IM $M_{M R L}$ measures evolve deterministically with respect to the average evolution of each component degradation process.

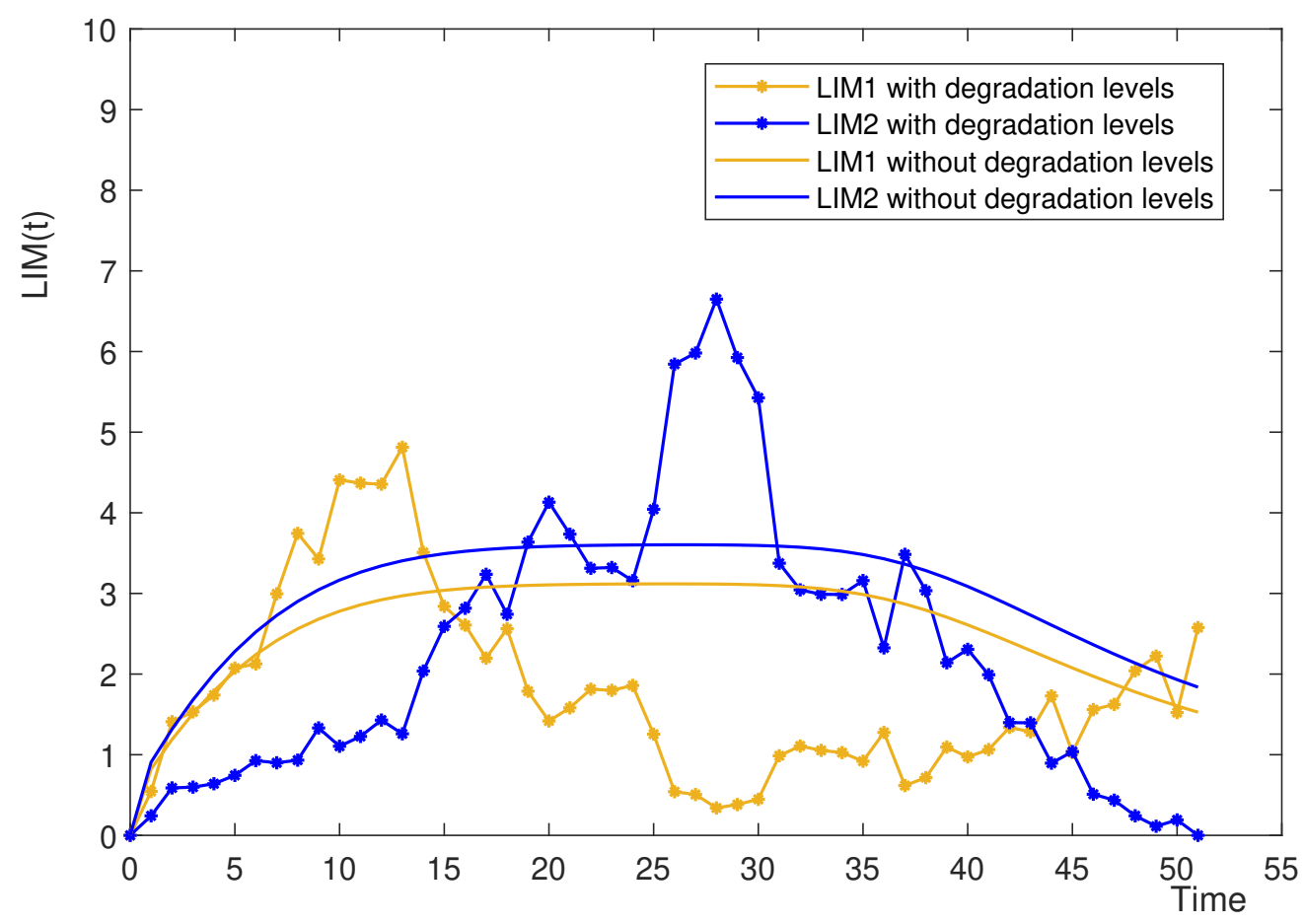

Figure 3: $I M_{M R L}$ measure of individual components with respect to time $t$ in two cases (Case 1 and Case 2a).

These experimental results show that $I M_{M R L}$ is time-dependent and both the information level of the components and their degradation levels are crucial for the $I M_{M R L}$ 's values and the associated components importance ranking.

\subsection{Link with existing importance measures}

In this section, the link between the proposed importance measure $I M_{M R L}$ with two existing importance measures, Birnbaum importance measure [2] and conditional reliability-based importance measure [10], is discussed to highlight the originality and the complementarity of the proposed $I M_{M R L}$ measure with respect to existing ones. In addition, constructing these links allows also evaluating one 
measure when other ones are known.

\subsubsection{Birnbaum importance measure}

It is shown [19] that the reliability of the system can be expressed as a linear function of the reliability of component $i$ as follows:

$$
R(t)=r^{i}(t) \cdot\left[R\left(t \mid \mathfrak{Z}_{t}^{i}=1\right)-R\left(t \mid \mathfrak{Z}_{t}^{i}=0\right)\right]+R\left(t \mid \mathfrak{Z}_{t}^{i}=0\right)
$$

where,

- $R\left(t \mid \mathfrak{Z}_{t}^{i}=1\right)$ is the probability that the system is still surviving at time $t$ when component $i$ is functioning at time $t$. For a parallel structure system, $R\left(t \mid \mathfrak{Z}_{t}^{i}=1\right)=1$;

- $R\left(t \mid \mathfrak{Z}_{t}^{i}=0\right)$ is the probability that the system is functioning at time $t$ given that component $i$ is in a failed state. $R\left(t \mid \mathfrak{Z}_{t}^{i}=0\right)=0$ if component $i$ is a critical one i.e., a failure of component $i$ leads to a failure of the whole system [19].

The Birnbaum' importance measure, that is defined as the partial differentiation of the system reliability with respect to the reliability of a component, is the most popular importance measure in ranking components for various decision-making purposes $[2,14,19]$.

From Eq.(15), Birnbaum's importance measure for component $i$ is mathematically defined as:

$$
I B^{i}(t)=\frac{\partial R(t)}{\partial r^{i}(t)}=R\left(t \mid \mathfrak{Z}_{t}^{i}=1\right)-R\left(t \mid \mathfrak{Z}_{t}^{i}=0\right) .
$$

By definition, $I B$ measure is bounded, i.e., $0 \leq I B^{i}(t) \leq 1$ with $i=1, . ., n$. The larger $I B^{i}(t)$, the bigger is the impact in the reliability of the system when the reliability of component $i$ changes. Therefore, $I B$ measure can be seen as a sensitivity importance measure [19].

Note also that $I B$ measure is time-dependent. If the system information is available at time $t, I B$ measure can be extended as follows:

$$
I B^{i}\left(t+u \mid \mathbf{Z}_{t}\right)=\frac{\partial R\left(t+u \mid \mathbf{Z}_{t}\right)}{\partial r^{i}\left(t+u \mid \mathfrak{Z}_{t}^{i}\right)}=R\left(t+u \mid \mathfrak{Z}_{t+u}^{i}=1, \mathbf{Z}_{t}\right)-R\left(t+u \mid \mathfrak{Z}_{t+u}^{i}=0, \mathbf{Z}_{t}\right) .
$$

An illustration of the evolution of $I B$ measure for a two-component system is shown in Figures 4 and 5 .

By using Eq.(15) and Eq.(17), the conditional reliability of the system at time $t+u$ given the condition/information of system components at time $t, \mathbf{Z}_{t}$, can be written as:

$$
R\left(t+u \mid \mathbf{Z}_{t}\right)=r^{i}\left(t+u \mid \mathbf{Z}_{t}\right) . I B^{i}\left(t+u \mid \mathbf{Z}_{t}\right)+R\left(t \mid \mathfrak{Z}_{t}^{i}=0\right) .
$$

From Eq.(13) and Eq.(18), we obtain a relationship between the proposed $I M_{M R L}$ measure and Birnbaum's measure as follows:

$$
I M_{M R L}^{i}(t)=\int_{0}^{\infty} I B^{i}\left(t+u \mid \mathbf{Z}_{t}\right) \cdot\left(r^{i}(u)-r^{i}\left(t+u \mid \mathfrak{Z}_{t}^{i}\right) d u\right.
$$

This allows evaluating $I M_{M R L}$ measure from Birnbaum's measure. 


\subsubsection{Conditional reliability-based importance measure}

Recently, an importance measure, namely conditional reliability-based importance measure, has been proposed for ranking components and preventive maintenance decision-making for a given mission [10]. Indeed, the condition reliability-based importance measure for a component $i$ is mathematically defined as follows:

$$
R I M^{i}(u, t)=R\left(t+u \mid \mathbf{Z}_{t}, \mathfrak{Z}_{t}^{i}=3\right)-R\left(t+u \mid \mathbf{Z}_{t}\right)
$$

By definition, RIM measure quantifies the potential improvement in the system reliability within the horizon $(t, t+u)$ if component $i$ is replaced at time $t[10]$ considering the available information related to the current states of all components at time $t$. Furthermore, RIM measure is time-dependent and bounded, i.e., $0 \leq R I M^{i}(u, t) \leq 1$ with $i=1, . ., n$. $R I M^{i}(u, t)=0$ means that the replacement of component $i$ at time $t$ does not lead to any change in the system reliability at time $t+u$. $R I M^{i}(0, t)=1$ if component $i$ is critical and it is in a failed state at time $t$.

It is also pointed out in [10], $R I M$ measure is an adequate measure for ranking components and preventive maintenance decision-making at time $t$ regarding to a given reliability level of the system at $t+u$. For further discussions, the interested reader can refer to [10].

From Eq.(13) and Eq.(20), we get a direct link between the proposed importance measure $I M_{M R L}$ and $R I M$ measure as follows:

$$
I M_{M R L}^{i}(t)=\int_{0}^{\infty} R I M^{i}(u, t) d u
$$

This relationship shows that $R I M^{i}(u, t)$ quantifies the impact on the system reliability at time $t+u$ when component $i$ is replaced at time $t$ while $I M_{M R L}^{i}(t)$ measures the impact of this replacement on the whole residual life time of the system, infinite horizon. This means that $R I M$ and $I M_{M R L}$ measures are complementary in ranking components and/or maintenance decision-making since both reliability and residual life time are two important reliability metrics of industrial systems [19].

Example 1 (continued) As an example, we reconsider the system of two components shown in Figure 1. The mathematical formulation of $I B, R I M$ and $I M_{M R L}$ measures for each component and the relationships between the three measures are reported in Table 2.

\begin{tabular}{c||c|c|c|}
\hline Component & $I B^{i}\left(t \mid \mathbf{Z}_{t}\right)$ & $R I M^{i}(u, t)$ & $I M_{M R L}^{i}(t)$ \\
\hline $\mathrm{C} 1$ & $r^{2}\left(t+u \mid \mathfrak{Z}_{t}^{2}\right)$ & $I B^{1}\left(t \mid \mathbf{Z}_{t}\right) \cdot\left(r^{1}(u)-r^{1}\left(t+u \mid \mathfrak{Z}_{t}^{1}\right)\right)$ & $\int_{0}^{\infty} R I M^{1}(u, t) d u$ \\
$\mathrm{C} 2$ & $r^{1}\left(t+u \mid \mathfrak{Z}_{t}^{1}\right)$ & $I B^{2}\left(t \mid \mathbf{Z}_{t}\right) \cdot\left(r^{2}(u)-r^{2}\left(t+u \mid \mathfrak{Z}_{t}^{2}\right)\right)$ & $\int_{0}^{\infty} R I M^{2}(u, t) d u$ \\
\hline
\end{tabular}

Table 2: $I B, R I M$ and $I M_{M R L}$ measures for a series structure system of two components.

Figure 4 sketches $I B$ and $R I M$ measures for each component at time $t+u$ given that two components are functioning at time $t=10$ but their degradation levels are unknown. The results show that, 
according to $I B$ measure or $R I M$ measure, When $30 \leq u \leq 50$, both $I B$ and $R I M$ measures lead to the same importance ranking, $C 2>C 1$. However, However, it should be pointed out that $R I M$ measure leads to a more contrasted decision for the ranking of both components. This can be explained by the fact that $R I M$ of a component allows considering the current condition of the component while $I B$ does not. However, when $u<30$ or $u>50$, both $I B$ and $R I M$ do not allow ranking the two components while the proposed $I M_{M R L}$ measure provides only one importance ranking, $C 2>C 1$, see again Table 1 (Case 1 ).
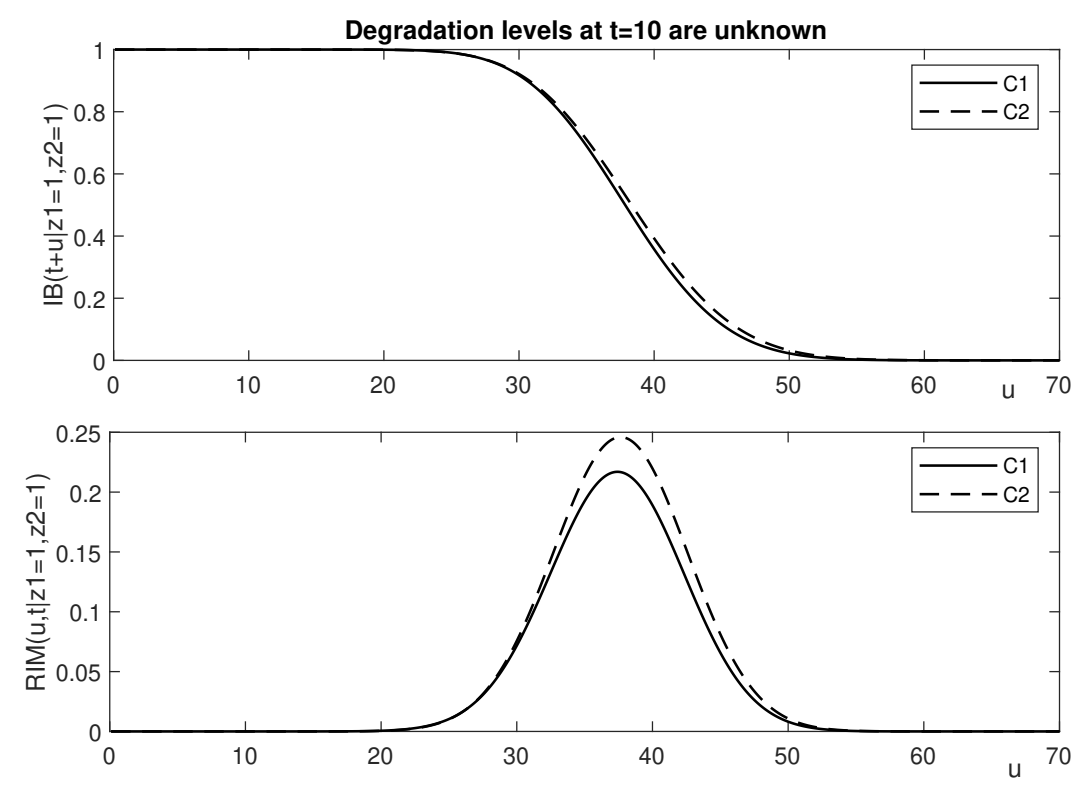

Figure 4: $I B$ and $R I M$ measures at time $t+u$ when the degradation levels at time $t=10$ are unknown.

In the same manner, we assume now the degradation levels of two components at time $t=10$ are measured. Figure 5 shows $I B$ and $R I M$ measures for each component at time $t+u$ given degradation levels of two components at time $t=10: X_{t}^{1}=21.76, X_{t}^{2}=12.82$ (see again Case 2a of Table 1). Similar results are herein obtained. Note however that, according to $I B$ or $R I M$ measure, when $25 \leq u \leq 55$, component $C 1$ is more important than $C 2(C 1>C 2)$. In addition, when $u<25$ or $u>55$ both $I B$ and $R I M$ do not allow clearly ranking the two components. When compared to the result given in Table 1 for this case, the proposed $I M_{M R L}$ measure provides only one importance ranking, $C 1>C 2$. 

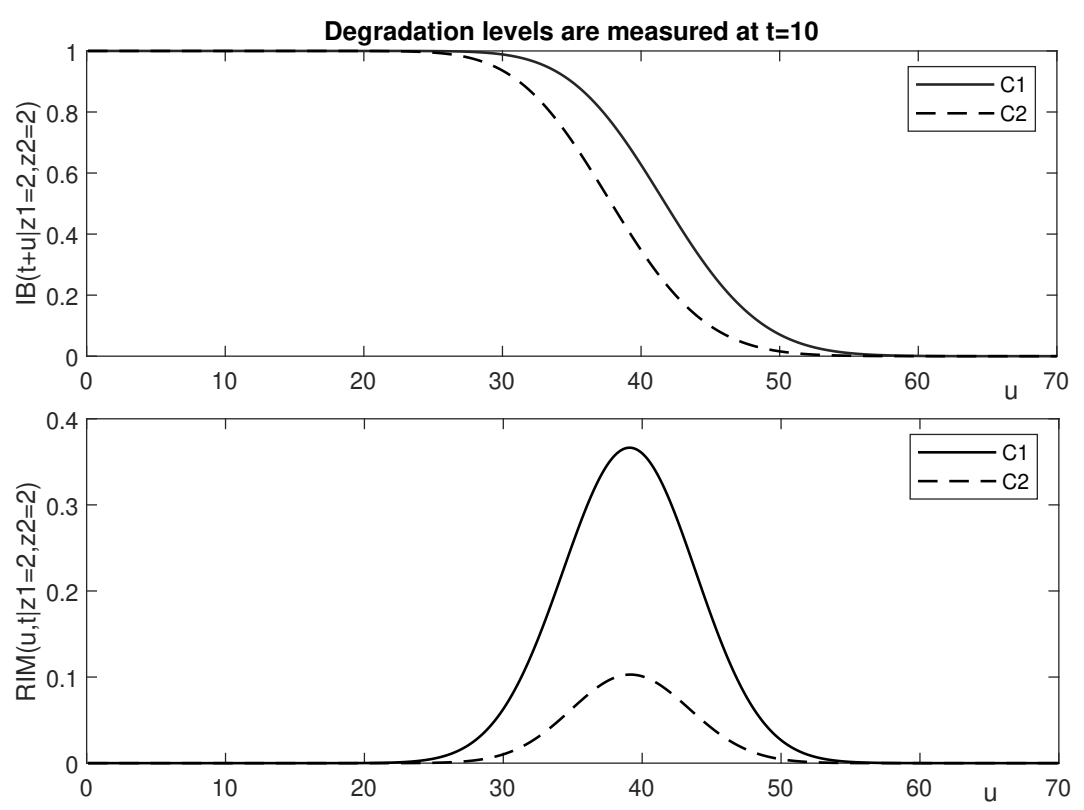

Figure 5: $I B$ and $R I M$ measures at time $t+u$ given the degradation levels at time $t=10$.

According the obtained results, it is pointed out that, in this example, $I M_{M R L}$ measure seems to be better than $I B$ and $R I M$ measures in ranking components since $I M_{M R L}$ leads to an unique components ranking for any value of $u$ while $I B$ and $R I M$ measures may lead to different components ranking with different values of $u$.

\section{3 $I M_{M R L}$ of a group of components}

The proposed $I M_{M R L}$ measure can also applied to a group of several components. Indeed, without loss of generality, we consider a group $G_{k}$ including $k$ components, denoted $\left\{j_{1}, . . j_{k}\right\}$ with $k=2,3, \ldots$, $I M_{M R L}$ measure of group $G_{k}$ at time $t$ can be mathematically defined as follows:

$$
I M_{M R L}^{\left\{j_{1}, . . j_{k}\right\}}(t)=M R L\left(t+u \mid \mathbf{Z}_{t},\left\{\mathfrak{Z}_{t}^{j_{1}}=3, \ldots, \mathfrak{Z}_{t}^{j_{k}}=3\right\}\right)-M R L\left(t \mid \mathbf{Z}_{t}\right),
$$

where $M R L\left(t+u \mid \mathbf{Z}_{t},\left\{\mathfrak{Z}_{t}^{j_{1}}=3, \ldots, \mathfrak{Z}_{t}^{j_{k}}=3\right\}\right)$ is the system mean residual life when $k$ components $\left\{j_{1}, . . j_{k}\right\}$ are jointly replaced at time $t$ considering the actual condition of the other components of the system.

According to the definition, $I M_{M R L}^{\left\{j_{1}, . . j k\right\}}(t)$ quantifies the residual life improvement of the system thanks to the joint replacement of group $G_{k}$ at time $t$. Note that if all components of the system are jointly replaced at time $t$, i.e., the system is renewed, the maximum improvement in the system life time is reached.

It should be noticed that that if components are structurally dependent, the joint replacement of several component can reduce the disassembly impacts on the failure/degradation processes of other components [8]. 
Due to joint effects in the system residual life time when group of components are replaced together, $I M_{M R L}$ is not additive, i.e.,

$$
I M_{M R L}^{\left\{j_{1}, \ldots j_{k}\right\}}(t) \neq \sum_{l=1}^{k} I M_{M R L}^{\left\{j_{1}\right\}}(t) .
$$

It is pointed out in [10] that, RIM measure can also be applied to a group of several components. Therefore, the direct link between the proposed $I M_{M R L}$ measure and $R I M$ can be extended as follows:

$$
I M_{M R L}^{\left\{j_{1}, . . j_{k}\right\}}(t)=\int_{0}^{\infty} R I M^{\left\{j_{1}, . . j_{k}\right\}}(u, t) d u
$$

The use and the advantages of $I M_{M R L}$ measure in ranking a component/group of components will be discussed in more detail in Section 5 .

\section{An extension of $I M_{M R L}$ measure for maintenance decision-making}

Maintenance cost and benefit from maintenance interventions are strongly interrelated and often plays a significant role in maintenance decision-making. Therefore, they should be considered in the components ranking process. To this aim, $I M_{M R L}$ measure is herein extended to incorporate both the benefit gained by the replacement of a component and its replacement cost. Indeed, the extension of $I M_{M R L}$ measure for a component $i$ at time $t$ is mathematically defined as follows:

$$
I M_{M R L-c}^{i}(t)=\frac{h\left(I M_{M R L}^{i}(t)\right)}{C^{i}},
$$

where:

- $h\left(I M_{M R L}^{i}(t)\right)$ is the benefit associated to the replacement of component $i$, which is considered as a function of the residual life improvement of the system thanks to the replacement of component $i$ at time $t, I M_{M R L}^{i}(t)$;

- $C^{i}$ is the replacement cost of component $i$ and can be divided into two parts: $C^{i}=c^{i}+c_{d} \cdot d^{i}$ where $c_{d} \cdot d^{i}$ is the downtime cost due to production loss during replacement of component $i$, that takes $d^{i}$ time units. Note that $c^{i}$ includes all other costs related to spare part, labour, set-up, etc.

The benefit function $h\left(I M_{M R L}^{i}(t)\right)$ may have different forms, i.e., a linear or non-linear function with respect to $I M_{M R L}^{i}(t)$. As an example, in this work a linear form is considered as follows:

$$
h\left(I M_{M R L}^{i}(t)\right)=Q \cdot I M_{M R L}^{i}(t),
$$

with $Q$ is positive number and can be seen as the benefit resulting in each operating time unit of the system. The two following cases can be specified for $Q$ :

- $Q=1$, i.e., $h\left(I M_{M R L}^{i}(t)\right)=I M_{M R L}^{i}(t)$; in this case, $I M_{M R L-C}^{i}(t)$ represents the ratio between the improvement in the system life time, gained from the replacement of component $i$ at time $t$, and the replacement cost of component $i$; 
- $Q>1, h\left(I M_{M R L}^{i}(t)\right)$ can be expressed as a benefit gained from the improvement in the system residual life that is provided by the replacement of component $i$ at time $t$. In such a way, $I M_{M R L-C}$ measure can assist in selecting the most cost-effective component for preventive maintenance regarding to a specific threshold $O(O \geq 1)$ for the benefit-cost. More precisely:

- if $I M_{M R L-C}^{i}(t) \geq O$, component $i$ is considered as cost-effective one at time $t$, i.e., component $i$ could be admissible for the preventive maintenance selection;

- $0 \leq I M_{M R L-C}^{i}(t)<O$ means that the cost benefit given by the replacement of component $i$ is not enough. Consequently, component $i$ is not cost-effective at time $t$, i.e., it should not be preventively replaced at time $t$ at least from a financial point of view.

It is worth noting that the value of $O$ may be determined from a technical or economical issue, e.g., maintenance optimisation phase.

Just like $I M_{M R L}$ measure, $I M_{M R L-C}$ can be applied for a group of several components as follows:

$$
I M_{M R L-C}^{\left\{j_{1}, . . j_{k}\right\}}(t, u)=\frac{h\left(I M_{M R L}^{\left\{j_{1}, . . j_{k}\right\}}(t, u)\right)}{C\left\{j_{1}, . . j_{k}\right\}},
$$

where $C^{\left\{j_{1}, . . j_{k}\right\}}$ is the total maintenance cost for the replacement of the group components $\left(j_{1}, . . j_{k}\right)$. It must be noticed that in a multi-component system, economic dependence, whereby the joint maintenance of several components is cheaper than when these components are separately maintained, often exists between components $[13,18]$. Indeed, it is pointed out in [9] that when several components are simultaneously replaced, the maintenance cost saving arises from the sharing of the replacement set-up cost and the reduction of replacement duration.

Therefore, when $k$ components $\left\{j_{1}, . . j_{k}\right\}$ are jointly replaced, the total cost saving can be defined as follows:

$$
C S^{\left\{j_{1}, . . j_{k}\right\}}=a \cdot \sum_{i=1}^{k} c^{i}+b \cdot c_{d} \cdot \sum_{i=1}^{k} d^{i}
$$

where:

- $a(0 \leq a)$ is the cost-saving factor for the joint replacement of $k$ components $\left\{j_{1}, . . j_{k}\right\}$. As it is pointed out in [23], the cost saving is commonly equal to $5 \%$ of the total maintenance cost of the components $(a=0.05)$;

- $b(0 \leq b)$ is the duration-saving factor for the joint replacement of $k$ components.

By definition, $a, b$ represent the economic dependence degree between components. The bigger are $a$ and $b$, the stronger is the economic dependence between the components. When $a=0$ and $b=0$, the components are economically independent. A detailed investigation on the impact of the economic dependence between components in condition maintenance decision-making process is discussed in $[9,23]$. 
The total maintenance cost when $k$ components are jointly replaced can be finally evaluated by:

$$
C^{\left\{j_{1}, . . j_{k}\right\}}=\sum_{i=1}^{k} C^{i}-C S^{\left\{j_{1}, . . j_{k}\right\}} .
$$

From an economical point of view, the $I M_{M R L-C}$ measure seems to be an adequate indicator for the preventive maintenance selection because it considers not only the benefit resulting from the system life time improvement given by the replacement of a group of components, the corresponding replacement cost but also the economic dependence between components. An illustration on the use of $I M_{M R L-C}$ measure for ranking components and preventive maintenance decision-making is discussed in the following section.

\section{$5 \quad$ Numerical example}

In this section, we show how the proposed $I M_{M R L}$ measure and its extension $I M_{M R L-C}$ can be used for ranking components/groups of components and preventive maintenance decision-making. For this purpose, a 4-non repairable component system whose reliability block diagram is given in Figure 6, is considered. It is important to note that proposed importance measure $I M_{M R L}$ and its extension $I M_{M R L-C}$ can be applied for any kind of systems for which a reliability structure function is available.

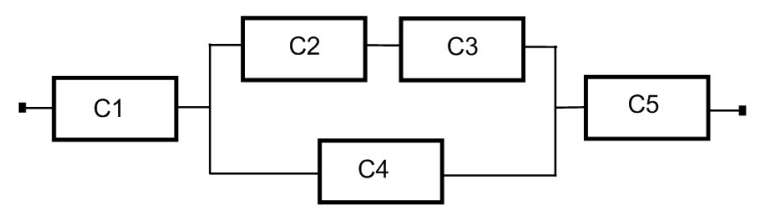

Figure 6: Example of a 4-component system.

From the system's reliability block diagram and the underlying structure function, the reliability of the system can be calculated from the system components' reliability as follows:

$$
R(t)=r^{1}(t) \cdot r^{2}(t)+r^{1}(t) \cdot r^{3}(t) \cdot r^{4}(t)-r^{1}(t) \cdot r^{2}(t) \cdot r^{3}(t) \cdot r^{4}(t)
$$

For components reliability modelling, both failure time laws and degradation process are considered. It is assumed that the failure behaviors of components C3 and $\mathrm{C} 4$ are described by Weibull ditribution with shape and scale parameters $\gamma^{i}, \eta^{i}(i=3,4)$, see Appendix A. For components $\mathrm{C} 1$ and $\mathrm{C} 2$, their reliability behaviors are assumed to be described by a degradation-threshold failure model with homogeneous gamma stochastic processes with shape and scale parameters $\alpha^{i}, \beta^{i}(i=1,2)$, see Appendix B. Component C1 or C2 fails when its degradation level searches a failure threshold $L^{1}$ or $L^{2}$ respectively. Table 3 reports the numerical values of $\alpha^{i}, \beta^{i}, L^{i}$ (with $i=1,2$ ) and $\gamma^{i}$, $\eta^{i}$ (with $i=3,4)$. Based on the failure/degradation process' parameters, the MTTF (mean time to failure) of each component is evaluated (see Table 3). Note that these parameters are in arbitrary time unit (atu). 


\begin{tabular}{c|cccccc}
\hline Unit & $\alpha^{i}$ & $\beta^{i}$ & $\gamma^{i}$ & $\eta^{i}$ & $L^{i}$ & $M T T F$ \\
\hline $\mathrm{C} 1$ & 1.3 & 1.6 & - & - & 100 & 48.69 \\
$\mathrm{C} 2$ & 1.4 & 1.5 & - & - & 100 & 47.96 \\
$\mathrm{C} 3$ & - & - & 65 & 2.2 & - & 58.33 \\
$\mathrm{C} 4$ & - & - & 60 & 1.5 & - & 54.03 \\
\hline
\end{tabular}

Table 3: Parameters of a 4-component system.

The residual life time of the system decreases due to the degradation of its components over time. To improve the system residual life time, one or several components should be preventively maintained at a certain time $t$. In that context, a challenging question for maintenance decision-making could be which component(s) should be chosen for preventive maintenance ? To answer this question, we propose here an heuristic decision rule based on $I M_{M R L}$ and/or $I M_{M R L-C}$ measure(s), i.e., the component/group of components having the highest importance ranking, according to $I M_{M R L}$ or/and $I M_{M R L-C}$ criterion, should be selected for preventive maintenance.

\section{$5.1 \quad I M_{M R L}$ measure and importance ranking}

It is considered in this section that the maintenance costs are not available due to whatever reason. $I M_{M R L}$ measure is applied to rank a component/group of components.

\subsection{1 $I M_{M R L}$ of individuals components}

At time $t=10$, the condition/state of each component is delivered by an inspection. Suppose that components $\mathrm{C} 3$ and $\mathrm{C} 4$ are in a working state, i.e., $\mathfrak{Z}_{t}^{3}=\mathfrak{Z}_{t}^{4}=1$. For components $\mathrm{C} 1$ and $\mathrm{C} 2$, two cases are considered:

- Case 1: Components C1 and C2 are working at time $t$ but their degradation levels are not measured due to a technical or economical reason, $\mathfrak{Z}_{t}^{1}=\mathfrak{Z}_{t}^{2}=1$. Based on the available information, the conditional reliability of each component is predicted. Then, $I M_{M R L}$ of each component is evaluated, the associated components ranking is then obtained according to $I M_{M R L}$ 's values. The results reported in Table 4 show that $\mathrm{C} 1$ is the most important component and the importance ranking is $\mathrm{C} 1>\mathrm{C} 2>\mathrm{C} 3>\mathrm{C} 4$. Therefore, if only one component can be preventively replaced at time $t$, component $\mathrm{C} 1$ should be selected.

- Case 2: Components $\mathrm{C} 1$ and $\mathrm{C} 2$ are working at time $t$ and their degradation levels are measured, i.e., $\mathfrak{Z}_{t}^{1}=\mathfrak{Z}_{t}^{2}=2$. For the degradation levels at time $t=10$, we reuse herein a numerical result presented in Section 3, i.e., $X_{t}^{1}=15.91$ and $X_{t}^{2}=22.16$. Then, the conditional reliability of components $\mathrm{C} 1$ and $\mathrm{C} 1$ are updated. The obtained results on $I M_{M R L}$ measure of each component and the components ranking are given in Table 4. There is evidence that 
the $I M_{M R L}$ 's value of each component changes when compared to the results in the previous case. Indeed, component $\mathrm{C} 1$ is no longer the most important component and the importance ranking becomes $\mathrm{C} 2>\mathrm{C} 1>\mathrm{C} 3>\mathrm{C} 4$. Consequently, for maintenance decision-making, component $\mathrm{C} 2$ should be, in this case, selected for preventive maintenance at time $t$. It is noteworthy that a different result on $I M_{M R L}$ measure could be obtained with different values of $X_{t}^{1}$ and $X_{t}^{2}$.

\begin{tabular}{c||c|c|c|c|c|c|c|c|}
\hline \multicolumn{1}{c||}{} & \multicolumn{3}{c|}{ Case 1: without degradation levels of C1 \& C2 } & \multicolumn{3}{c|}{ Case2 :with degradation levels of C1 \& C2 } \\
\cline { 2 - 8 } Unit & $\mathfrak{Z}_{t}^{i}$ & $X_{t}^{i}$ & $I M_{M R L}^{i}$ & Ranking & $\mathfrak{J}_{t}^{i}$ & $X_{t}^{i}$ & $I M_{M R L}^{i}$ & Ranking \\
\hline C1 & 1 & - & 4.34 & 1 & 2 & 15.91 & 2.70 & 2 \\
C2 & 1 & - & 2.05 & 2 & 2 & 22.16 & 2.86 & 1 \\
C3 & 1 & - & 0.23 & 3 & 1 & - & 0.32 & 3 \\
C4 & 1 & - & 0.17 & 4 & 1 & - & 0.23 & 4 \\
\hline
\end{tabular}

Table 4: $I M_{M R L}$ measure and importance ranking at time $t=10$.

The obtained results point out that the information about the components' states at time $t$ is crucial on $I M_{M R L}$ measure and, as a consequence, on the components ranking. The absence of the degradation levels of components may lead to an inaccurate importance ranking.

In all studies presented the remainder of the paper, only the case 2 is considered, i.e., at time $t=10, \mathfrak{Z}_{t}^{1}=\mathfrak{Z}_{t}^{2}=2, X_{t}^{1}=15.91, X_{t}^{2}=22.16 ; \mathfrak{Z}_{t}^{3}=\mathfrak{Z}_{t}^{4}=1$.

As a comparison study, Birnbaum measure and RIM measure are applied for each individual component. The obtained results are sketched in Figure 7. It can be seen again that both $I B$ and $R I M$ of each component change with time. Consequently, the components ranking may change. This is especially true with $R I M$ measure. Furthermore, the importance ranking provided by $I B$ measure is not the same as those given by $R I M$ measure or the proposed $I M_{M R L}$ one. More precisely, when $u<44, R I M$ provides the same the importance ranking given by $I M_{M R L}$. This is no longer true when $u \geq 44$. These differences can be explained by the fact that $I B$ measure of a given component does not allow considering the current condition/state of the component while both $R I M$ and $I M_{M R L}$ do. In addition, $R I M$ is evaluated with an finite horizon while $I M_{M R L}$ is defined with an infinite horizon. 

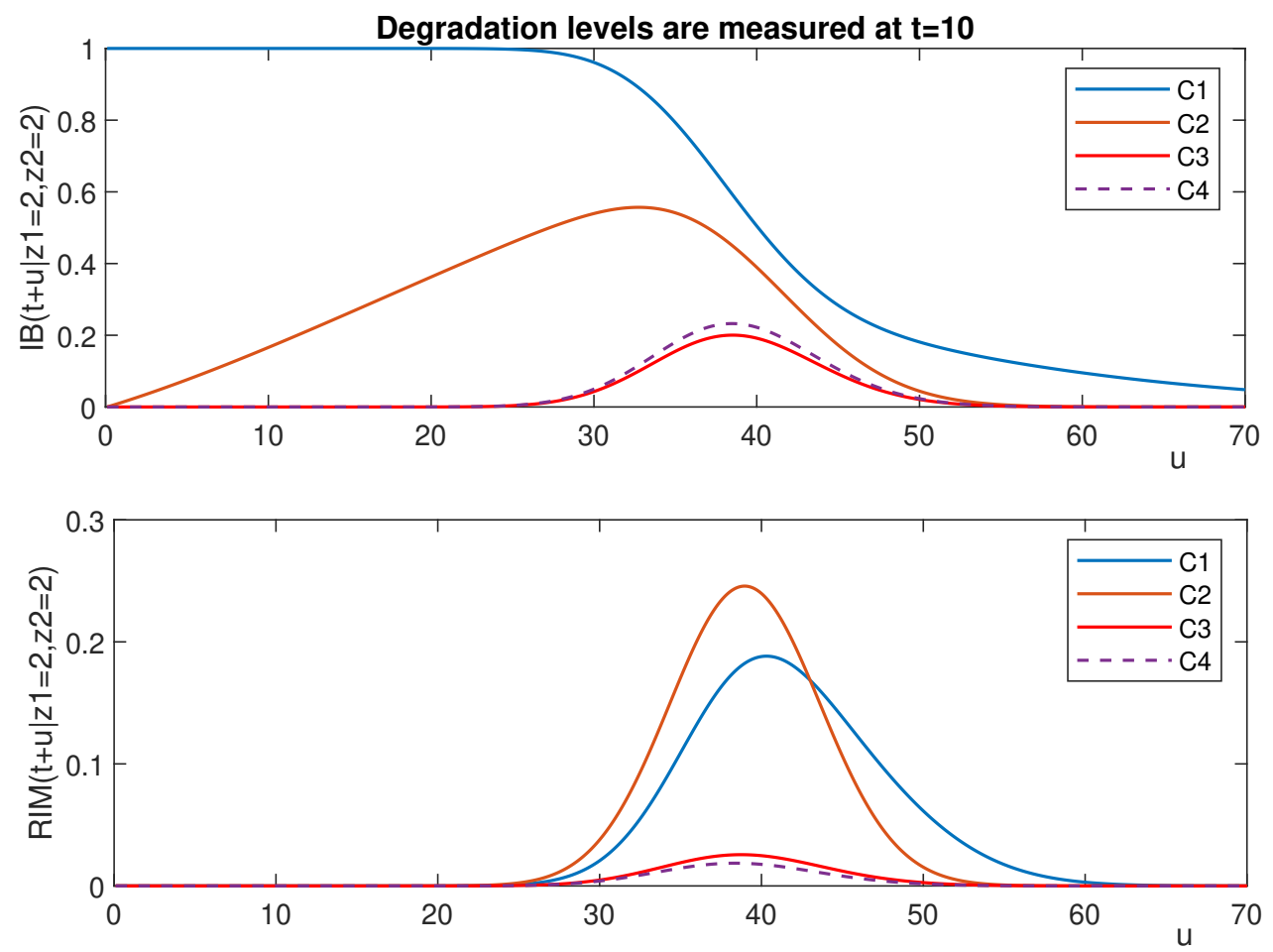

Figure 7: $I B$ and $R I M$ measures at time $t+u$ given information available at time $t=10$.

\subsubsection{I $I M_{M R L}$ of groups of components}

To illustrate the use of the proposed $I M_{M R L}$ measure for groups of components, $I M_{M R L}$ measure is herein applied to two-component groups given the available information on components at time $t=10$, which are presented in the previous section (see case 2). Of course, $I M_{M R L}$ can be implemented to three-component groups or four-component groups in a similar way.

Table 5 reports the obtained results of $I M_{M R L}$ for two-component groups.

\begin{tabular}{|c||c|c|}
\hline \multicolumn{1}{|c||}{} & \multicolumn{2}{c|}{ At time $t=10: \mathfrak{Z}_{t}^{1}=\mathfrak{Z}_{t}^{2}=2, X_{t}^{1}=15.91, X_{t}^{2}=22.16 ; \mathfrak{Z}_{t}^{3}=\mathfrak{Z}_{t}^{4}=1$} \\
\cline { 2 - 3 } Group & $I M_{M R L}^{G}(t)$ & Ranking \\
\hline$(\mathrm{C} 1, \mathrm{C} 2)$ & 8.24 & 1 \\
$(\mathrm{C} 1, \mathrm{C} 3)$ & 3.37 & 2 \\
$(\mathrm{C} 1, \mathrm{C} 4)$ & 3.17 & 3 \\
$(\mathrm{C} 2, \mathrm{C} 3)$ & 2.91 & 4 \\
$(\mathrm{C} 2, \mathrm{C} 4)$ & 2.89 & 5 \\
$(\mathrm{C} 3, \mathrm{C} 4)$ & 0.60 & 6 \\
\hline
\end{tabular}

Table 5: $I M_{M R L}$ measure and 2-component groups ranking.

The results shows that group $(\mathrm{C} 1, \mathrm{C} 2)$ having the highest $I M_{M R L}$ value is the most important group and the less important one is group $(\mathrm{C} 3, \mathrm{C} 4)$.

As $R I M$ measure can be extended for groups of several components, $R I M$ measure is herein applied to two-component groups. The results in Figure 8 show again that the importance ranking 
associated with RIM measure may change with time. In addition, it is difficult to point out the groups ranking when $u<26$ or $u>60$.

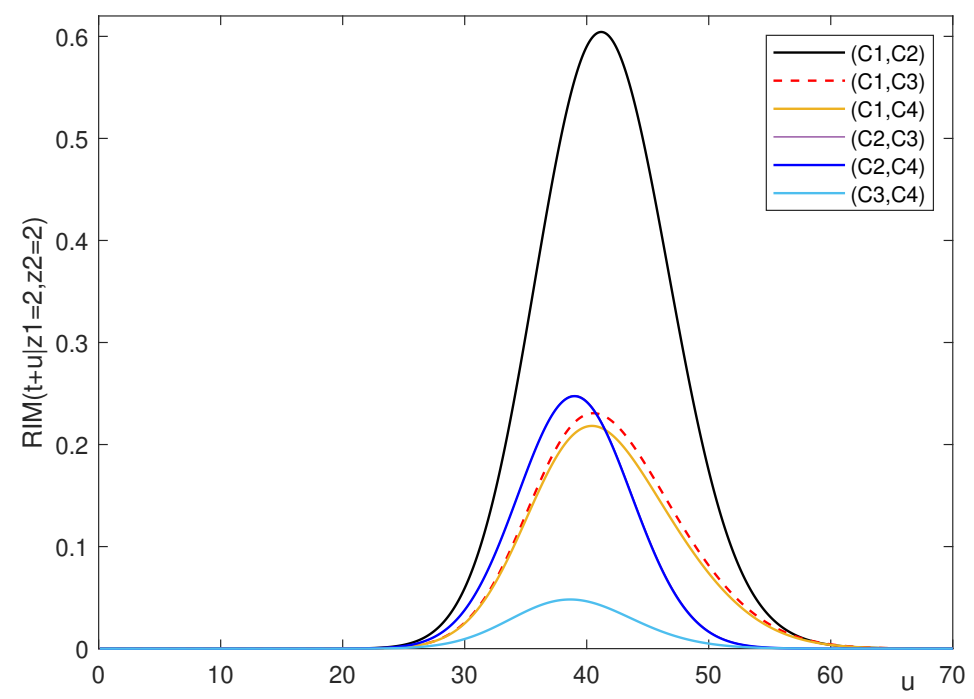

Figure 8: RIM measures at time $t+u$ given information available at time $t=10$.

\section{2 $I M_{M R L-C}$ measure for importance ranking and selective maintenance}

We suppose now the maintenance costs are known: $c^{1}=150, c^{2}=250, c^{3}=50, c p^{4}=80$ and $c_{d}=20$ acu (arbitrary cost unit). The components' maintenance duration are $d^{1}=5, d^{2}=3, d^{3}=5, d^{4}=3$ atu. The economic dependence factors are $a=5 \%, b=10 \%$. The improvement in the system life time thanks to the replacement of a component $i$, is herein converted into an economic benefit with benefit rate $B=500$. In order to consider both the economic benefit and the maintenance costs in ranking a component or a group of components, $I M_{M R L-C}$ measure, is herein used.

\subsection{1 $I M_{M R L-C}$ of individual components}

Table 6 reports the results of $I M_{M R L-C}$ measure applied for each individual component.

\begin{tabular}{c||c|c|c|c|}
\hline \multicolumn{1}{c||}{} & \multicolumn{4}{c|}{ Degradation levels measured at $t=10$} \\
\cline { 2 - 5 } Unit & $\mathfrak{Z}_{t}^{i}$ & $X_{t}^{i}$ & $I M_{M R L-C}^{i}$ & Ranking \\
\hline C1 & 2 & 15.91 & 5.39 & 1 \\
C2 & 2 & 22.16 & 4.62 & 2 \\
C3 & 1 & - & 1.07 & 3 \\
C4 & 1 & - & 0.83 & 4 \\
\hline
\end{tabular}

Table 6: $I M_{M R L-C}$ measure and components ranking.

It is apparent that the $I M_{M R L-C}$ measure leads to an importance ranking that is not the same 
as the one provided by $I M_{M R L}$ (see again Table 4, case 2). Furthermore, according to $I M_{M R L}$ criteria, component $\mathrm{C} 2$ is the most important one while, regarding to $I M_{M R L-C}$ measure, $\mathrm{C} 1$ is the most important component. This is because $I M_{M R L}$ focuses only on the improvement of the system residual life, i.e., the maintenance cost is not considered, while $I M_{M R L-C}$ considers both the benefit associated with the system life time improvement and the related maintenance cost.

\subsection{2 $I M_{M R L-C}$ of groups of components}

$I M_{M R L-C}$ measure is now applied for all groups of two components and the obtained results are shown in Table 7.

\begin{tabular}{|c||c|c|}
\hline \multicolumn{1}{|c||}{} & \multicolumn{2}{c|}{ At time $t=10: \mathfrak{Z}_{t}^{1}=\mathfrak{Z}_{t}^{2}=2, X_{t}^{1}=15.91, X_{t}^{2}=22.16 ; \mathfrak{Z}_{t}^{3}=\mathfrak{Z}_{t}^{4}=1$} \\
\cline { 2 - 3 } Group & $I M_{M R L-C}^{G}(t)$ & Ranking \\
\hline (C1,C2) & 7.86 & 1 \\
$(\mathrm{C} 1, \mathrm{C} 3)$ & 4.56 & 2 \\
$(\mathrm{C} 1, \mathrm{C} 4)$ & 4.37 & 3 \\
$(\mathrm{C} 2, \mathrm{C} 3)$ & 3.39 & 5 \\
$(\mathrm{C} 2, \mathrm{C} 4)$ & 3.44 & 4 \\
$(\mathrm{C} 3, \mathrm{C} 4)$ & 1.32 & 6 \\
\hline
\end{tabular}

Table 7: $I M_{M R L-C}$ measure and 2-component groups ranking with $a=5 \%, b=10 \%$.

It is not surprising again that the two-component groups ranking provided by $I M_{M R L-C}$ measure is not the same as the one given by $I M_{M R L}$, see again Table 5. More precisely, according to $I M_{M R L}$ measure, the groups ranking is $(\mathrm{C} 1, \mathrm{C} 2)>(\mathrm{C} 1, \mathrm{C} 3)>(\mathrm{C} 1, \mathrm{C} 4)>(\mathrm{C} 2, \mathrm{C} 3)>(\mathrm{C} 2, \mathrm{C} 4)>(\mathrm{C} 3, \mathrm{C} 4)$ while $I M_{M R L-C}$ leads to another importance ranking: $(\mathrm{C} 1, \mathrm{C} 2)>(\mathrm{C} 1, \mathrm{C} 3)>(\mathrm{C} 1, \mathrm{C} 4)>(\mathrm{C} 2, \mathrm{C} 4)>(\mathrm{C} 2, \mathrm{C} 3)>(\mathrm{C} 3, \mathrm{C} 4)$.

\subsubsection{Impact of economic dependence on ranking groups of components}

To study the impact of economic dependence in $I M_{M R L-C}$ and the associated importance ranking, we change the economic dependence degree between component. More precisely, it is assumed that the duration-saving factor is equal to $30 \%(b=30 \%)$. The obtained results are reported in Table 8 .

\begin{tabular}{|c||c|c|}
\hline \multicolumn{1}{|c||}{} & \multicolumn{2}{c|}{ At time $t=10: \mathfrak{Z}_{t}^{1}=\mathfrak{Z}_{t}^{2}=2, X_{t}^{1}=15.91, X_{t}^{2}=22.16 ; \mathfrak{Z}_{t}^{3}=\mathfrak{Z}_{t}^{4}=1$} \\
\cline { 2 - 3 } Group & $I M_{M R L-C}^{G}(t)$ & Ranking \\
\hline$(\mathrm{C} 1, \mathrm{C} 2)$ & 8.37 & 1 \\
$(\mathrm{C} 1, \mathrm{C} 3)$ & 5.11 & 2 \\
$(\mathrm{C} 1, \mathrm{C} 4)$ & 4.78 & 3 \\
$(\mathrm{C} 2, \mathrm{C} 3)$ & 3.67 & $\mathbf{4}$ \\
$(\mathrm{C} 2, \mathrm{C} 4)$ & 3.64 & $\mathbf{5}$ \\
$(\mathrm{C} 3, \mathrm{C} 4)$ & 1.28 & 6 \\
\hline
\end{tabular}

Table 8: $I M_{M R L-C}$ measure and ranking of 2-component groups with $a=5 \%, b=30 \%$. 
It is shown that, when compared to the results reported in Table $7, I M_{M R L-C}$ values change and a new groups ranking appears. This means that the economic dependence degree between components has an important impact on ranking groups of components.

\subsection{Joint consideration of $I M_{M R L}$ and $I M_{M R L-C}$ for importance rankings}

In this section, both $I M_{M R L}$ and $I M_{M R L-C}$ measures are jointly considered in finding the most relevant component/group of components for preventive maintenance decision-making.

Figure 9 sketches the $I M_{M R L}$ with respect to $I M_{M R L-C}$ of individual component, evaluated at time $t=10$. Note that the degradation levels of $\mathrm{C} 1$ and $\mathrm{C} 2$ at time $t=10$ are measured, i.e., $\mathfrak{Z}_{t}^{1}=\mathfrak{Z}_{t}^{2}=2, X_{t}^{1}=15.91, X_{t}^{2}=22.16$ and $\mathfrak{Z}_{t}^{3}=\mathfrak{Z}_{t}^{4}=1$.

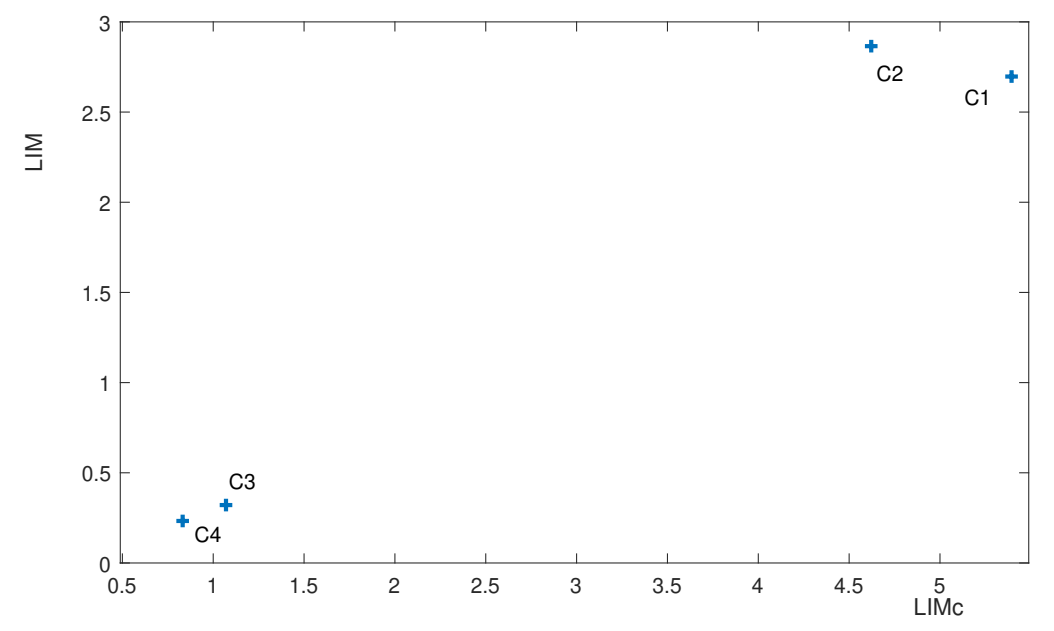

Figure 9: Joint consideration of $I M_{M R L}$ and $I M_{M R L-C}$ for individual components ranking.

Note that the less important components are provided in the bottom left corner the figure and the components in the top right corner are the most important ones. Obviously, each measure leads to a different importance ranking and it is difficult to identify the most important component in absolute terms. Nevertheless, if the decision maker considers that the improvement in the system life time is a priority criterion, component $\mathrm{C} 2$ should be selected for preventive maintenance while $\mathrm{C} 1$ should be chosen if an improvement of 2.70 atu in the system residual life is enough.

In a similar way, Figure 10 sketches the $I M_{M R L}$ vs $I M_{M R L-C}$ of two-component groups, evaluated with $a=5 \%, b=30 \%$. The results show that $(\mathrm{C} 1, \mathrm{C} 2)$ is the most important group according to both $I M_{M R L}$ and $I M_{M R L-C}$ measures. This means that group $(\mathrm{C} 1, \mathrm{C} 2)$ should be maintained together at time $t=10$. 


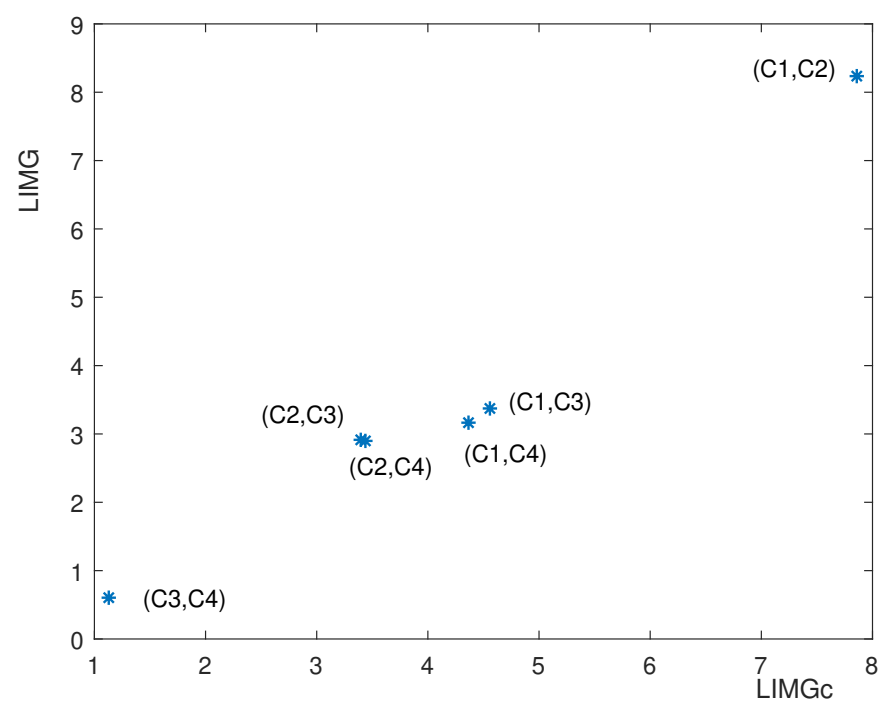

Figure 10: Joint consideration of $I M_{M R L}$ and $I M_{M R L-C}$ for ranking two-component groups.

\section{Conclusions}

In this work, a residual life-based importance measure, namely $I M_{M R L}$, defined as the improvement ability in the system life time when replacing a component or a group of several components, is introduced. The proposed $I M_{M R L}$ measure can help to rank a component/group of components according to its importance in the system residual life improvement by considering not only the available information related to the current condition (state or/and degradation level) of all components at a given time point but also the system structure into a singe technical metric. The links with existing importance measures (Birnbaum's importance measure and conditional reliability-based importance measure) are also established and discussed to highlight the originality and complementarity of the proposed $I M_{M R L}$ measure. Furthermore, to consider economic aspects (e.g., economic dependence between components, preventive/corrective maintenance costs and the benefit gained from maintenance operations), an extension of $I M_{M R L}$ measure is then developed. Thereby, $I M_{M R L-C}$ can be used in finding the most cost-effective component/group of several components. To illustrate the use and advantages of the proposed measures, a numerical example of a 4-non repairable component system is investigated. The numerical results reveals that, at a given time point, $I M_{M R L}$ and $I M_{M R L-C}$ depend strongly on the components degradation/failure process and the available information levels on the current condition of the components. In addition, the economic dependence degree has also an important impact on ranking groups of component. Importantly, $I M_{M R L}$ and $I M_{M R L-C}$ may lead to two different importance rankings. Nevertheless, from a practical point of view, IMMRL and $I M_{M R L-C}$ measures are complementary and should be jointly considered in finding the most appropriate component/group of components to be preventively maintained. 
This paper presents the development of our research in the framework of importance measures for predictive maintenance decision-making presented in part in [11]. Our future research will focus on the development of the proposed importance measures for joint inspection and maintenance decisionmaking with imperfect inspection quality. Another perspective could be a comparison study with others importance measures for components ranking and maintenance decision-making of complex systems.

\section{Appendix}

\section{Appendix A. Reliability assessment with Weibull lifetime distribution}

If the failure behavior of a component is described by a Weibull law, its lifetime follows a Weibull distribution, $f_{\tau^{i}}(t)$, given by

$$
f_{\tau^{i}}(t)=\frac{\gamma^{i}}{\eta^{i}}\left(\frac{t}{\eta^{i}}\right)^{\left(\gamma^{i}-1\right)} e^{-\left(\frac{t}{\eta^{i}}\right)^{\gamma^{i}}}
$$

with parameters $\gamma^{i}>0$ (shape or form parameter) and $\eta^{i}>0$ (scale parameter). The reliability of the component $i$ is expressed as

$$
r^{i}(t)=e^{\left(\frac{t}{\eta^{i}}\right)^{\gamma^{i}}}
$$

If component $i$ is working at time $t$, i.e., $\mathfrak{Z}_{t}^{i}=1$, the conditional reliability of component $i$ is written as

$$
r^{i}\left(t+u \mid \mathfrak{Z}_{t}^{i}=1\right)=e^{\left[\left(\frac{t+u}{\eta^{i}}\right)^{\gamma^{i}}-\left(\frac{t}{\eta^{i}}\right)^{\gamma^{i}}\right]} .
$$

\section{Appendix B. Reliability assessment with gamma degradation process}

It is assumed that the degradation behavior of component $i$ is described by a gamma processes $\left(\tilde{X}_{t}\right)_{t \geq 0}$ with the following characteristics:

- $\left(\tilde{X}_{t}\right)_{t \geq 0}$ has independent increments;

- for all $0 \leq l<t$, the random increment $\tilde{X}_{t}-\tilde{X}_{l}$ follows a gamma probability density function (pdf) with shape parameter $\alpha^{i}(t-l)$ and scale parameter $\beta^{i}$ :

$$
f_{X_{t}^{i}}(x)=\frac{1}{\Gamma\left(\alpha^{i}(t-l)\right)}\left(\beta^{i}\right)^{\alpha^{i}(t-l)} x^{\alpha^{i}(t-l)-1} e^{-\beta^{i} x} \mathcal{I}_{\{x \geq 0\}}
$$

where, $\mathcal{I}_{\{.\}}$is an indicator function. $\mathcal{I}_{\{x\}}=1$ if $x \geq 0, \mathcal{I}_{\{x\}}=0$ and otherwise;

The mean degradation speed is $\alpha^{i} / \eta_{i}$ and its variance is $\alpha^{i} /\left(\beta^{i}\right)^{2}$. Noting that various degradation behaviors can be modeled by changing the couple of parameters $\alpha^{i}, \beta^{i}$.

According to the degradation process, the reliability of a component $i$ can be assessed by:

$$
r^{i}(t)=\mathbb{P}\left(X_{t}^{i}<L^{i}\right)=\int_{0}^{L^{i}} \frac{1}{\Gamma\left(\alpha^{i} t\right)}\left(\beta^{i}\right)^{\alpha^{i} t} x^{\alpha^{i} t-1} e^{-\beta^{i} x} d x .
$$


If component $i$ is working at time $t$ and the degradation level of component $i$ is measured at time $t$, $\mathfrak{Z}_{t}^{i}=2, X_{t}^{i}=x_{t}^{i}\left(x_{t}^{i}<L^{i}\right)$, the conditional reliability of component $i$ can be evaluated by:

$$
r^{i}\left(t+u \mid \mathfrak{Z}_{t}^{i}=2\right)=\int_{x_{t}^{i}}^{L^{i}} \frac{1}{\Gamma\left(\alpha^{i} u\right)}\left(\beta^{i}\right)^{\alpha^{i} u} x^{\alpha^{i} u-1} e^{-\beta^{i} x} d x,
$$

\section{References}

[1] S. Beeson and J.D. Andrews. Importance measures for non-coherent-system analysis. IEEE Transactions on Reliability, 52(3):301-310, 2013.

[2] L.W. Birnbaum. On the importance of different elements in a multielement system. multivariate analysis. Academic Press, 2, 1969.

[3] E. Borgonovo. Differential importance and comparative statics: An application to inventory management. International Journal of Production Economics, 111(1):170-179, 2008.

[4] E. Borgonovo, H. Aliee, Glaß M., and J. Teich. A new time-independent reliability importance measure. European Journal of Operational Research, 254(2):427-442, 2016.

[5] E. Borgonovo and G.-E. Apostolakis. A new importance measure for risk-informed decision making. Reliability Engineering and System Safety, 72(2):193-212, 2001.

[6] C.-S. Byington, M.-J. Roemer, and T. Galie. Prognostic enhancements to diagnostic systems for improved condition-based maintenance. In Proceedings, IEEE aerospace conference, volume 6, pages 6-16. IEEE, 2002.

[7] M.C. Cheok, G.W. Parry, and R.R. Sherry. Use of importance measures in risk informed regulatory applications. Reliability Engineering and System Safety, 60(3):213-226, 1998.

[8] D.-H. Dinh, P. Do, and B. Iung. Degradation modeling and reliability assessment for a multicomponent system with structural dependence. Computers $\&$ Industrial Engineering, 144:106443, 2020 .

[9] P. Do, R. Assaf, and B. Scarf, P.and Iung. Modelling and application of condition-based maintenance for a two-component system with stochastic and economic dependencies. Reliability Engineering \& System Safety, 182:86-97, 2019.

[10] P. Do and C. Bérenguer. Conditional reliability-based importance measures. Reliability Engineering \& System Safety, 193:106633, 2020.

[11] P. Do, C. Bérenguer, and E. Borgonovo. MRL-based importance measures. In Proc. of 29th European Safety and Reliability Conference - ESREL 2019, pages 1229-1235, 2019. 
[12] P. Do, A. Voisin, E. Levrat, and B. Iung. Remaining useful life (rul) based maintenance decision making for deteriorating systems. In 2nd IFAC Workshop on Advanced Maintenance Engineering, Service and Technology (A-Mest'12), 22-23 November, Sevilla, Spain, 2012, page 8pp, 2012.

[13] J.S. Hong. Joint reliability importance of k-out-of-n systems. European Journal of Operational Research, 142:539-547, 2002.

[14] W. Kuo and X. Zhu. Importance Measures in Reliability, Risk, and Optimization: Principles and Applications. John Wiley and Sons, 2012- 384 pages.

[15] X. Lu, P. Baraldi, and E. Zio. A data-driven framework for identifying important components in complex systems. Reliability Engineering \& System Safety, 204:107197, 2020.

[16] K.-A. Nguyen, P. Do, and A. Grall. Condition-based maintenance for multi-component systems using importance measure and predictive information. International Journal of Systems Science: Operations \& Logistics, 1(4):228-45, 2014.

[17] K.-A. Nguyen, P. Do, and A. Grall. Joint predictive maintenance and inventory strategy for multicomponent systems using birnbaum's structural importance. Reliability Engineering 6 S System Safety, 168:249-261, 2017.

[18] R.P. Nicolai and R. Dekker. Optimal maintenance of multi-component systems: A review. In K.A.H. Kobbacy and D.N.P. Murthy, editors, Complex System Maintenance Handbook, Springer Series in Reliability Engineering, pages 263-286. Springer, London, 2008.

[19] M. Rausand and H. Hoyland. System Reliability Theory - Models, Statistical methods and Application. Wiley Series in Probability and Statistics. Wiley Interscience, second edition, 2004.

[20] J.M. Van Noortwijk. A survey of the application of Gamma processes in maintenance. Reliability Engineering and System Safety, 94:2-21, 2009.

[21] H.-C. Vu, P. Do, and Barros. A stationary grouping maintenance strategy using mean residual life and the birnbaum importance measure for complex structures. IEEE Transactions on Reliability, 65(1):217-234, 2016.

[22] H.-C. Vu, P. Do, and A. Barros. (MRL, T) grouping policy for complex structure systems. In Advance in Safety, Reliability and Risk Management, Proc.ESREL 2013, 29 September - 2 October Amsterdam, Netherlands. Taylor and Francis 8p, 2013.

[23] R.E Wildeman, R. Dekker, and A.C.J.M. Smit. A dynamic policy for grouping maintenance activities. European Journal of Operational Research, 99:530-551, 1997. 
[24] S. Wu, Y. Chen, Q. Wu, and Z. Wang. Linking component importance to optimisation of preventive maintenance policy. Reliability Engineering and System Safety, 146:26-32, 2016.

[25] T. Xia, Y. Dong, L. Xiao, S. Du, E. Pan, and L. Xi. Recent advances in prognostics and health management for advanced manufacturing paradigms. Reliability Engineering 85 System Safety, 178:255-268, 2018.

[26] M. Zhang. A heuristic policy for maintaining multiple multi-state systems. Reliability Engineering E System Safety, 203:107081, 2020. 